\title{
Aptitude à la coagulation de laits préemprésurés secs
}

\author{
par \\ Suwana ROONGTANAPIROM*, \\ M. BENNASAR et B. TARODO DE LA FUENTE \\ Laboratoire de Technologie alimentaire \\ Université des Sciences et Techniques du Languedoc \\ place E.-Bataillon - 34060 Montpellier cedex
}

\section{INTRODUCTION}

Le $\mathrm{XX}^{\mathrm{e}}$ congrès international de laiterie qui s'est déroulé à Paris en juin 1978 a mis en évidence la place importante que les laits reconstitués sont en train de prendre dans l'industrie laitière. Importance qui va se traduire par la publication prochaine d'une monographie par la Fédération Internationale de Laiterie [1].

Ce produit présente en effet un intérêt économique et nutritionnel évident pour les pays en voie de développement comme l'a souligné le docteur Farkhondeh [2] lors de sa conférence sur la reconstitution et la recombinaison des laits et des produits laitiers.

Parmi les divers produits préparés à partir de laits reconstitués, les fromages sont particulièrement intéressants grâce à leur relative facilité d'acceptation par les populations de ces pays, ce qui n'est pas toujours le cas du lait à cause de la déficience en lactase souvent observée chez les adultes [3].

Dans ce domaine, beaucoup de progrès ont été accomplis en France et une mise au point intéressante en a été faite au cours du séminaire [4] qui s'est déroulé à l'Ecole Nationale d'Industrie Laitière de Mamirolle dans les jours qui ont suivi le $\mathrm{XX}^{\mathrm{e}}$ congrès.

Les premiers essais de préparation de fromages à partir de laits reconstitués ont mis en œuvre les procédés classiques de fabrication. Lablée [5] a démontré qu'il était possible de fabriquer n'importe quel type de fromage en adaptant les procédés traditionnels aux caractères particuliers de ces matières premières, à condition cependant, de n'utiliser que des poudres de lait de qualité supérieure du point de

\footnotetext{
* Adresse actuelle : Université de Chulalongkorn (Thaïlande).
} 
vue bactériologique et n'ayant subi qu'un traitement thermique modéré.

L'adaptation de la méthode de fabrication en continu StenneHutin permit ensuite de résoudre le problème du manque de cohésion du caillé obtenu par les procédés classiques et surtout de mettre en œuvre des poudres de qualité alimentaire standard [6].

Le procédé MMV d'ultrafiltration en fromagerie mis au point par Maubois, Mocquot et Vassal a lui aussi été appliqué dès 1973 aux laits reconstitués $[7,8]$. Maubois, lors du $\mathrm{XX}^{\mathrm{e}}$ congrès en a précisé l'intérêt pour le développement de l'aide alimentaire au tiersmonde [9]. La tendance actuelle est à l'utilisation du pouvoir séparateur des membranes pour préparer un lait concentré qui sera ensuite séché par atomisation. La poudre obtenue peut alors être stockée, transportée et finalement réhydratée dans l'exacte proportion du fromage à obtenir avant d'être emprésurée, ce qui supprime les opérations d'égouttage, de pressage et les problèmes posés par l'utilisation du sérum. Cette méthode permettrait en outre de réduire les pertes importantes de matières azotées dans le lactosérum, inévitables lors de la fabrication traditionnelle.

Nous avons poursuivi les mêmes objectifs en emprésurant le lait à basse température comme l'ont indiqué Maubois, Mocquot et Vassal [7], en utilisant des quantités de présure normales et en maintenant l'action de l'enzyme pendant un temps suffisant pour que la phase enzymatique spécifique soit terminée, avant de le sécher par atomisation. Les poudres ainsi préparées coagulent après reconstitution avec une quantité d'eau que l'on peut réduire à la teneur en eau du fromage à obtenir.

La composition et le comportement des poudres préemprésurées dépendant des traitements technologiques subis par le lait, nos recherches ont porté sur des laits écrémés crus ou pasteurisés, normaux ou concentrés par évaporation ou ultrafiltration. Dans chaque cas, nous avons déterminé les conditions de préemprésurage, les caractéristiques physico-chimiques des poudres préparées et leur aptitude à la coagulation.

Nous avons montré également qu'il était possible de provoquer la coagulation d'un lait normal par addition de poudre préemprésurée et d'obtenir un coagulum de bonne qualité.

\section{MATERIELS ET METHODES}

Laits: nous avons utilisé des laits de mélange crus ou pasteurisés et des laits reconstitués à partir de poudre Spray de qualité bas chauffage pour fromagerie. 
Concentration: les laits de mélange ont été concentrés deux et trois fois soit par évaporation d'eau sous vide, soit par ultrafiltration. Dans le premier cas, l'opération est conduite pendant $1 \mathrm{~h}$ environ dans un évaporateur à plaques APV Junior de telle sorte que la température du produit ne dépasse jamais $40^{\circ} \mathrm{C}$.

Dans le deuxième cas, nous avons utilisé un appareil RhônePoulenc, modèle Pléiade UF-P7, équipé de membranes IRIS 3042 dont le seuil de séparation correspond à un poids moléculaire de 20000 . L'ultrafiltration est conduite à $18^{\circ} \mathrm{C}$ pendant une durée maximale de $8 \mathrm{~h}$; dans le cas du lait cru il s'ensuit normalement une légère diminution du $\mathrm{pH}$.

Séchage : la transformation en poudre des laits étudiés est réalisée dans un sécheur pilote Anhydro. Les laits préemprésurés sont atomisés à basse température $\left(10^{\circ} \mathrm{C}\right)$ grâce à une turbine tournant à 22400 tours par minute. La température de l'air chaud est maintenue à $160^{\circ} \mathrm{C}$ et le débit de lait réglé pour que la température de la poudre n'excède jamais $90^{\circ} \mathrm{C}$.

Présure : nous avons utilisé une présure animale en poudre (Maret) de force initiale 270 U.P./g selon la définition de l'Unité Présure de Berridge [10].

L'emprésurage est effectué avec une solution enzymatique à 5 p. 100 dans un tampon phosphate de $\mathrm{pH} 6,66$ à $25^{\circ} \mathrm{C}$, préparée $24 \mathrm{~h}$ à l'avance et conservée à $+4^{\circ} \mathrm{C}$.

La mesure des temps de floculation est réalisée d'après la méthode de Sommer et Matsen [11].

L'activité de l'enzyme est déterminée par mesure de la libération des substances azotées non protéiques (NPN) en fonction du temps, selon la technique indiquée par Alais [12].

Aptitude à la coagulation des laits préemprésurés secs : l'aptitude à la coagulation des laits préemprésurés secs et les propriétés mécaniques des coagulums qu'ils permettent d'obtenir sont étudiées à l'aide d'un thrombélastographe Hellige type C modifié. La méthode utilisée dérive de celle qui a été mise au point par Frentz et al, [13, 14, 15, 16] : le lait reconstitué à $4^{\circ} \mathrm{C}$ est placé dans la cuvette de mesure maintenue à la même température. L'enregistrement est mis en route en même temps que le réchauffement de la cuvette.

Dans ces conditions, le paramètre $r$ mesure le temps écoulé depuis le début de l'enregistrement jusqu'au dédoublement du tracé thrombélastographique; $\mathrm{k}$ est le temps nécessaire pour que l'écartement du tracé atteigne $20 \mathrm{~mm}$, il représente la vitesse de raffermissement du gel ; Am mesure l'écartement maximal des deux branches du tracé et correspond donc à la fermeté maximale du gel avant le début de sa synérèse. La figure 1 montre le schéma d'un thrombélastogramme avec ses différentes caractéristiques. 
Le rendement fromager est évalué dans les conditions suivantes inspirées des essais réalisés par Lelièvre [17] : le lait préparé est réchauffé à $40^{\circ} \mathrm{C}$, coagulé et maintenu à cette température jusqu'au début de la synérèse. Il est alors placé sur filtre en papier et laissé à température ambiante pendant un temps variable selon son origine. Le sérum recueilli est pesé et analysé.

Azote et calcium : les teneurs en azote et en calcium sont évaluées respectivement par les méthodes classiques de micro-Kjeldhal [12] et de spectrophotométrie d'absorption atomique [18].

Matière sèche et humidité : la matière sèche (ESD) est déterminée par évaporation au bain-marie et à l'étuve à $100 \pm 2^{\circ} \mathrm{C}$, tandis que l'humidité des laits secs est mesurée à l'aide d'un humidimètre "Moisture balance Cenco ».

Solubilité : la solubilité des poudres est testée selon la méthode de l'ADMI [19] en opérant à $0^{\circ} \mathrm{C}$ pour éviter la coagulation au moment de la reconstitution.

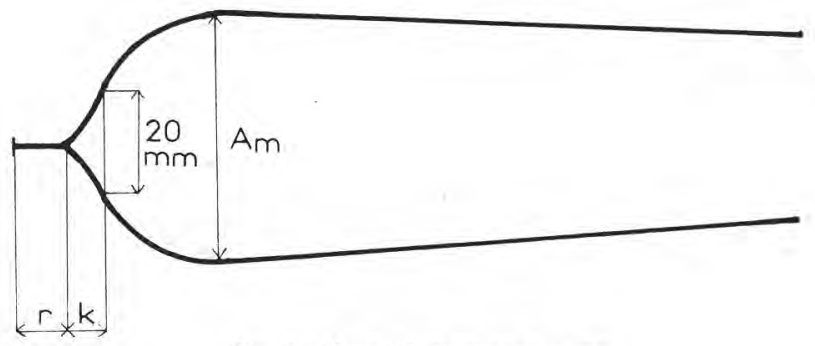

fig. 1 : thrombélastogramme

\section{RESULTATS}

\section{III.1. Etude des paramètres de préemprésurage}

Notre objectif était de déterminer les conditions de préemprésurage (quantité de présure, température et concentration en calcium) nécessaires et suffisantes pour que le temps de préemprésurage soit aussi court que possible et qu'il n'y ait aucun risque de floculation au moment du séchage.

Quantité de présure : nous avons emprésuré à $30^{\circ} \mathrm{C}$ des laits reconstitués à 9 et 27 p.100 de matières sèches et mesuré leur temps de floculation et la libération de NPN correspondante. Les résultats consignés dans le tableau 1 montrent une variation normale du temps 
TABLEAU 1. - Action de la présure sur les laits reconstitués à $30^{\circ} \mathrm{C}$

\begin{tabular}{|c|c|c|c|c|c|c|}
\hline \multirow{2}{*}{$\begin{array}{c}\text { Matières } \\
\text { sèches } \\
\text { p. } 100\end{array}$} & \multirow{2}{*}{$\begin{array}{c}\text { Taux } \\
\text { d'emprésu- } \\
\text { rage } \\
\text { (solution à } \\
5 \text { p. } 100 \text { ) } \\
\text { p. } 100\end{array}$} & \multirow{2}{*}{$\begin{array}{l}\text { Temps de } \\
\text { floculation } \\
\text { mn: sec }\end{array}$} & \multicolumn{4}{|c|}{ NPN libéré } \\
\hline & & & $\begin{array}{c}\text { départ } \\
\mathrm{mg} / \mathrm{l}\end{array}$ & $\begin{array}{l}\text { floculation } \\
\mathrm{mg} / \mathrm{l}\end{array}$ & $\begin{array}{l}\text { écart } \\
\mathrm{mg} / 1\end{array}$ & p. 100 \\
\hline 9 & 0,5 & $15: 45$ & 262 & 318 & 56 & 17,6 \\
\hline 9 & 1,5 & $10: 15$ & 266 & 321 & 55 & 17,1 \\
\hline 27 & 0,5 & $8: 25$ & 746 & 860 & 114 & 13,3 \\
\hline 27 & 1,5 & $3: 45$ & 746 & 861 & 115 & 13,4 \\
\hline
\end{tabular}

de floculation en fonction de la quantité d'enzyme utilisée ainsi que l'importance considérable de la concentration. Nous pouvons constater également qu'un lait concentré trois fois et emprésuré à 0,5 p. 100 coagule plus vite qu'un lait normal ayant reçu une dose triple d'enzyme.

De plus, la concentration en enzyme ne joue aucun rôle sur la quantité totale de NPN libérée à la fin de la phase primaire ; mais celle-ci est relativement moins importante dans le lait concentré que dans le lait normal.

Température de préemprésurage: les laits crus normaux (CN) et concentrés deux et trois fois par évaporation (CEV) ou ultrafiltration (CUF) ont été emprésurés avec 0,5 p. 100 de solution enzymatique à des températures de 5,10 et $15^{\circ} \mathrm{C}$. La mesure du temps de floculation de prélèvements aliquotes effectués à intervalles réguliers et portés à $40^{\circ} \mathrm{C}$ permet de déterminer la durée de préemprésurage minimale nécessaire à l'achèvement de la phase enzymatique primaire (tab. 2) [20].

Nous pouvons constater que le temps de préemprésurage minimal a tendance à diminuer lorsque la concentration des laits augmente, surtout si celle-ci est obtenue par ultrafiltration. Sa diminution est beaucoup plus nette avec l'accroissement de la température. A $15^{\circ} \mathrm{C}$, sauf dans le cas du lait normal, la floculation se produit spontanément après quelques minutes d'emprésurage. 


\section{TABLEAU 2}

Variations du temps de préemprésurage minimal en fonction de la température et de la nature des laits

\begin{tabular}{|c|c|c|c|}
\hline \multirow{2}{*}{ Laits } & \multicolumn{3}{|c|}{ Durée minimale de préemprésurage (mn) } \\
\hline & $5^{\circ} \mathrm{C}$ & $10^{\circ} \mathrm{C}$ & $15^{\circ} \mathrm{C}$ \\
\hline $\mathrm{CN}$ & 45 & 25 & 20 \\
\hline CEV 2 & 45 & 25 & $20 *$ \\
\hline CEV 3 & 40 & 25 & $20 *$ \\
\hline CUF 2 & 30 & 20 & $15^{*}$ \\
\hline CUF 3 & 30 & 20 & $15^{*}$ \\
\hline
\end{tabular}

* Début de floculation.

Influence de la concentration en calcium : bien que ce sel n'intervienne pas directement sur le déroulement de la phase primaire, nous avons étudié son action sur le temps de préemprésurage minimal nécessaire en ajoutant des quantités croissantes de $\mathrm{Ca} \mathrm{Cl}_{2}$ dans un lait reconstitué à 9 p. 100 de poudre.

Nous avons observé une diminution de la durée minimale de préemprésurage à $10^{\circ} \mathrm{C}$ avec l'accroissement de la teneur en calcium comme l'indiquent les valeurs du tableau 3.

\section{TABLEAU 3}

Action du calcium sur le temps de préemprésurage minimal à $10^{\circ} \mathrm{C}$

\begin{tabular}{c|c|c|c|c}
\hline Taux calcium (g/100 1 lait) & 0 & 10 & 20 & 30 \\
\hline Durée minimale préemprésurage (mn) & 65 & 55 & 40 & 30 \\
\hline
\end{tabular}




\section{III.2. Caractères physicochimiques des poudres de laits préemprésurés}

Les différentes poudres étudiées ont été obtenues à partir de laits crus $(\mathrm{C})$ et pasteurisés $(\mathrm{P})$ dont la composition et la durée minimale de préemprésurage figurent dans le tableau 4. Tous ces laits ont été emprésurés à $10^{\circ} \mathrm{C}$ avec $0,5 \mathrm{p} .100$ de solution de présure et l'action enzymatique a été poursuivie pendant $30 \mathrm{mn}$ après les temps minima indiqués dans le tableau 4.

L'aspect et les caractéristiques des laits secs obtenus concordent généralement avec ceux des poudres Sprays classiques (tab 5). C'est ainsi que les laits normaux donnent des produits fins, très légers, mais difficilement mouillables et très peu solubles, alors que les laits concentrés conduisent à des poudres plus grossières, plus denses, mais plus solubles.

L'absence de données pour la solubilité des laits secs concentrés par ultrafiltration provient d'une floculation partielle au cours de la mesure due à une plus grande instabilité certainement liée au $\mathrm{pH}$ très bas des laits de fabrication (tab. 4).

\section{TABLEAU 4}

Propriétés et temps de préemprésurage minimal des différents types de lait

\begin{tabular}{|c|c|c|c|c|c|}
\hline Laits & $\mathrm{pH}$ & $\begin{array}{c}\text { ESD } \\
\text { (p. 100) }\end{array}$ & $\begin{array}{c}N \text { total } \\
(\mathrm{g} / \mathrm{l})\end{array}$ & $\begin{array}{c}\text { Ca total } \\
(\mathrm{g} / \mathrm{l})\end{array}$ & $\begin{array}{c}\text { Durée } \\
\text { minimale } \\
\text { préemprésurage } \\
(\mathrm{mn})\end{array}$ \\
\hline $\mathrm{PN}$ & 6,70 & 8,6 & 5,0 & 1,07 & 50 \\
\hline PEV 2 & 6,62 & 18,0 & 10,3 & 2,46 & 45 \\
\hline PEV 3 & 6,50 & 26,3 & 15,4 & 3,63 & 45 \\
\hline $\mathrm{CN}$ & 6,45 & 9,5 & 5,4 & 1,23 & 25 \\
\hline CEV 2 & 6,42 & 18,3 & 11,0 & 2,54 & 25 \\
\hline CEV 3 & 6,40 & 29,5 & 16,6 & 3,96 & 25 \\
\hline CUF 2 & 6,10 & 10,4 & 7,4 & 1,53 & 20 \\
\hline CUF 3 & 5,98 & 13,1 & 10,3 & 2,05 & 20 \\
\hline
\end{tabular}




\section{TABLEAU 5}

Aspect et caractéristiques des poudres de lait

\begin{tabular}{|c|c|c|c|c|c|c|}
\hline \multirow{2}{*}{$\begin{array}{l}\text { Laits } \\
\text { de } \\
\text { départ }\end{array}$} & \multicolumn{6}{|c|}{ Poudres } \\
\hline & Aspect & $\begin{array}{c}\mathrm{N} \\
(\mathrm{mg} / \mathrm{g})\end{array}$ & $\begin{array}{c}\mathrm{Ca} \\
(\mathrm{mg} / \mathrm{g})\end{array}$ & $\mathrm{Ca} / \mathrm{N}$ & $\begin{array}{l}\text { Humidité } \\
\text { (p. 100) }\end{array}$ & $\begin{array}{l}\text { Solubilité } \\
\text { (p. 100) }\end{array}$ \\
\hline $\mathrm{PN}$ & Fine, très légère & 47,3 & 14,0 & 0,30 & 5,6 & 63 \\
\hline PEV 2 & $\begin{array}{l}\text { Légèrement agglomérée } \\
\text { Légère }\end{array}$ & 49,7 & 14,0 & 0,28 & 5,6 & 99 \\
\hline PEV 3 & $\begin{array}{l}\text { Légèrement sableuse } \\
\text { Légère }\end{array}$ & 51,5 & 13,4 & 0,26 & 3,6 & 99 \\
\hline $\mathrm{CN}$ & Fine très légère & 51,8 & 10,8 & 0,21 & 4,3 & 88 \\
\hline CEV 2 & $\begin{array}{l}\text { Plus grossière } \\
\text { Plus dense }\end{array}$ & 53,4 & 12,6 & 0,24 & 4,1 & 98 \\
\hline CEV 3 & $\begin{array}{l}\text { Plus grossière } \\
\text { Plus dense }\end{array}$ & 54,4 & 13,3 & 0,24 & 4,1 & 99 \\
\hline CUF 2 & Assez fine, dense & 70,1 & 13,6 & 0,20 & 4,6 & n.m.* \\
\hline CUF 3 & Assez fine, très dense & 82,1 & 15,0 & 0,18 & 5,4 & n.m.* \\
\hline
\end{tabular}

La nature des traitements technologiques de concentration du lait semble intervenir sur l'humidité des poudres correspondantes. On peut remarquer en effet que la teneur en eau a tendance à augmenter avec la concentration par ultrafiltration et à diminuer avec la concentration par évaporation.

La teneur en azote des laits secs augmente régulièrement avec la concentration du lait de fabrication cru ou pasteurisé ; cette augmentation semble être liée en partie à la variation d'humidité des poudres. Dans le cas des laits concentrés par ultrafiltration, l'accroissement de la teneur en azote des laits secs résulte du procédé de concentration utilisé. Le taux de calcium évolue parallèlement à celui de l'azote dans les laits crus et en sens contraire dans le cas des laits pasteurisés. 
TABLEAU 6

Variations des paramètres $\mathrm{r}$ et $\mathrm{k}$ en fonction du taux de reconstitution pour les laits pasteurisés

\begin{tabular}{|c|c|c|c|c|c|c|c|c|}
\hline \multirow{3}{*}{$\begin{array}{l}\text { Laits } \\
\text { de } \\
\text { départ }\end{array}$} & \multicolumn{8}{|c|}{ Taux de reconstitution (p. 100) } \\
\hline & \multicolumn{2}{|c|}{10} & \multicolumn{2}{|c|}{16,7} & \multicolumn{2}{|c|}{20} & \multicolumn{2}{|c|}{25} \\
\hline & $\begin{array}{c}\mathrm{r} \\
\mathrm{mn}: \mathrm{sec}\end{array}$ & $\begin{array}{c}\mathbf{k} \\
\mathrm{mn}: \mathrm{sec}\end{array}$ & $\begin{array}{c}\mathrm{r} \\
\mathrm{mn}: \mathrm{sec}\end{array}$ & $\begin{array}{c}\mathrm{k} \\
\mathrm{mn}: \mathrm{sec}\end{array}$ & $\begin{array}{c}\mathrm{r} \\
\mathrm{mn}: \mathrm{sec}\end{array}$ & $\begin{array}{c}\mathrm{k} \\
\mathrm{mn}: \mathrm{sec}\end{array}$ & $\begin{array}{c}\mathrm{r} \\
\mathrm{mn}: \mathrm{sec}\end{array}$ & $\begin{array}{c}\mathbf{k} \\
\mathrm{mn}: \mathrm{sec}\end{array}$ \\
\hline PN & $30: 03$ & $60: 00$ & $21: 21$ & $21: 54$ & f.i. $*$ & nm.** & f.i.* & $\mathrm{nm} . * *$ \\
\hline PEV 2 & $47: 12$ & $60: 00$ & $26: 33$ & 13:45 & $1: 30$ & $1: 36$ & $0: 51$ & $0: 42$ \\
\hline PEV 3 & $77: 30$ & $38: 45$ & $62: 30$ & $9: 15$ & 3:06 & $3: 21$ & $1: 48$ & $1: 30$ \\
\hline $\begin{array}{l}{ }^{*} \text { f.i. : } \\
* * \text { n.m. : }\end{array}$ & $\begin{array}{l}\text { oculatic } \\
\text { on mest }\end{array}$ & $\begin{array}{l}\text { instant } \\
\text { rable. }\end{array}$ & anée. & & & & & \\
\hline
\end{tabular}

\section{III.3. Aptitude à la coagulation des poudres}

L'aptitude à la coagulation et les propriétés physicochimiques des caillés préparés ont été étudiées pour les différents types de laits reconstitués à des taux variables.

\section{III.3.1. Floculation et coagulation}

Dans tous les cas où la mesure a pu être faite, les thrombélastogrammes conduisent à des résultats conformes aux tracés caractéristiques des divers types de laits indiqués dans les études précédentes $[15,16]$. Les laits reconstitués à partir de poudres issues de laits crus non concentrés floculent instantanément et rendent impossible tout enregistrement thrombélastographique. Dans le cas des laits pasteurisés et crus concentrés par évaporation, les temps de floculation ( $r$ ) et de raffermissement du coagulum (k) diminuent avec l'augmentation du taux de reconstitution. Le tableau 6 montre les résultats obtenus à partir de laits pasteurisés.

\section{III.3.2. Rendements}

Les rendements en poids de caillé frais, en azote et en calcium, ainsi que le rapport $\mathrm{Ca} / \mathrm{N}$ du caillé sont mesurés après égouttage. Les résultats de ces analyses effectuées dans les conditions fixées au chapitre "Matériel et méthodes » sont consignés dans le tableau 7. 
TABLEAU 7

Caractéristiques de l'égouttage des caillés en fonction du taux de reconstitution des différentes poudres de lait

\begin{tabular}{|c|c|c|c|c|c|c|c|c|c|c|c|c|c|c|c|c|}
\hline Lait initial & \multicolumn{2}{|c|}{$\mathrm{PN}$} & \multicolumn{2}{|c|}{ PEV 2} & \multicolumn{2}{|c|}{ PEV 3} & \multicolumn{2}{|c|}{$\mathrm{CN}$} & \multicolumn{2}{|c|}{ CEV 2} & \multicolumn{2}{|c|}{ CEV 3} & \multicolumn{2}{|c|}{ CUF 2} & \multicolumn{2}{|c|}{ CUF 3} \\
\hline $\begin{array}{l}\text { Taux de reconstitution } \\
\text { (p. 100) }\end{array}$ & 16,7 & 20 & 16,7 & 20 & 16,7 & 20 & 16,7 & 20 & 16,7 & 20 & 16,7 & 20 & 16,7 & 20 & 16,7 & 20 \\
\hline $\begin{array}{l}\text { Rendement en caillé } \\
\text { (p. 100) }\end{array}$ & 60,9 & 74,1 & 77,3 & 84,8 & 85,5 & 89,1 & 48,5 & 52,5 & 78,3 & 83,4 & 65,8 & 77,0 & 56,7 & 68,6 & 51,6 & 61,3 \\
\hline $\begin{array}{l}\text { Rendement en azote } \\
\text { (p. 100) }\end{array}$ & 91,2 & 94,8 & 95,4 & 97,2 & 96,6 & 98,0 & 85,5 & 86,5 & 94,4 & 96,3 & 90,3 & 93,4 & 88,9 & 91,5 & 86,3 & 88,6 \\
\hline $\begin{array}{l}\text { Rendement en calcium } \\
\text { (p. 100) }\end{array}$ & 91,8 & 95,2 & 94,0 & 96,4 & 95,8 & 97,1 & 87,7 & 88,8 & 95,1 & 96,5 & 93,0 & 95,5 & 69,4 & 81,3 & 74,4 & 79,9 \\
\hline $\begin{array}{l}\text { Sérum recueilli par rap- } \\
\text { port à l'eau de reconsti- } \\
\text { tution (p. 100) }\end{array}$ & 46,9 & 32,2 & 27,3 & 19,0 & 17,4 & 13,6 & 62,0 & 59,4 & 26,0 & 20,8 & 41,0 & 28,7 & 52,0 & 39,3 & 58,0 & 48,4 \\
\hline $\mathrm{Ca} / \mathrm{N}$ du caillé & 0,30 & 0,30 & 0,28 & 0,28 & 0,26 & 0,26 & 0,22 & 0,22 & 0,24 & 0,24 & 0,25 & 0,25 & 0,15 & 0,17 & 0,16 & 0,17 \\
\hline
\end{tabular}


Nous constatons que les rendements en caillé, azote et calcium augmentent avec le taux de reconstitution parallèlement à une meilleure rétention d'eau, comme l'indique la mesure des volumes de sérum recueillis. L'absence de variation du rapport $\mathrm{Ca} / \mathrm{N}$ en fonction de la teneur en matières sèches des laits reconstitués traduit d'autre part une évolution parallèle de ces deux éléments, sauf dans les laits ultrafiltrés où l'on peut constater qu'il augmente légèrement.

De manière générale, ces rendements augmentent également avec la concentration des laits mis en œuvre; cependant, dans le cas des laits crus concentrés trois fois, cette règle semble ne pas être suivie. Nous pouvons remarquer également que l'augmentation est beaucoup moins sensible dans les laits ultrafiltrés et qu'il y a même une diminution du rendement en calcium. Les variations du rapport $\mathrm{Ca} / \mathrm{N}$ sont en accord avec les valeurs déjà observées dans les poudres (tab. 5) : il augmente avec la concentration initiale des laits crus et diminue dans le cas des laits pasteurisés.

\section{3.3. Influence du calcium}

Nous avons testé l'influence d'un apport de calcium sur la floculation et la coagulation dans le cas de lait reconstitué à 20 p. 100 de poudre préparée à partir d'un lait pasteurisé concentré trois fois par évaporation. Les effets obtenus correspondent à ceux que l'on peut observer dans le cas d'un lait en poudre normal [15] : diminution des paramètres $\mathrm{r}$ et $\mathrm{k}$ et augmentation de l'amplitude du tracé thrombélastographique $(\mathrm{Am})$ indiquant un accroissement de la fermeté du gel.

L'addition de quantités croissantes de calcium dans des laits reconstitués à 20 p. 100 à partir des poudres des différents laits pasteurisés provoque d'autre part une diminution des rendements en caillé et en calcium, plus marquée cependant pour le premier. Le rendement azoté varie peu tandis que le rapport $\mathrm{Ca} / \mathrm{N}$ augmente régulièrement traduisant une insolubilisation du calcium ajouté (tab. 8).

\section{III.4. Utilisation des poudres comme agents de coagulation}

Nous avons réalisé à $0^{\circ} \mathrm{C}$ des mélanges à partir de lait en poudre Spray de qualité "bas chauffage " additionné de 10,8 p.100 de lait préemprésuré sec de différentes origines.

L'aptitude à la coagulation de ces mélanges a été étudiée par thrombélastographie et comparée à celle du lait en poudre Spray de qualité «bas chauffage » utilisé dans le mélange. Les résultats correspondants sont indiqués dans le tableau 9. Nous constatons dans tous les cas que la coagulation a lieu dans de meilleures conditions qu'avec le lait témoin comme le prouve la diminution des temps de floculation (r) et de formation du coagulum (k). Nous avons observé également une fermeté plus importante dans les gels provenant des mélanges. 
TABLEAU 8

Influence de l'addition de calcium sur l'égouttage et la composition des caillés de laits pasteurisés

\begin{tabular}{|c|c|c|c|c|c|c|c|c|c|c|c|c|}
\hline Lait initial & \multicolumn{4}{|c|}{$\mathrm{PN}$} & \multicolumn{4}{|c|}{ PEV 2} & \multicolumn{4}{|c|}{ PEV 3} \\
\hline $\begin{array}{l}\text { Taux de } \mathrm{CaCl}_{2} \\
(\mathrm{~g} / 100 \mathrm{l})\end{array}$ & 0 & 7,2 & 14,4 & 21,6 & 0 & 7,2 & 14,4 & 21,6 & 0 & 7,2 & 14,4 & 21,6 \\
\hline $\begin{array}{l}\text { Rendement en caillé } \\
\text { (p. 100) }\end{array}$ & 74,1 & 72,2 & 67,4 & 64,8 & 84,8 & 73,2 & 75,2 & 68,9 & 89,1 & 78,4 & 84,5 & 67,4 \\
\hline $\begin{array}{l}\text { Rendement en azote } \\
\text { (p. 100) }\end{array}$ & 94,8 & 94,9 & 94,3 & 93,8 & 97,2 & 95,5 & 96,6 & 94,6 & 98,0 & 96,2 & 97,4 & 94,6 \\
\hline $\begin{array}{l}\text { Rendement en calcium } \\
\text { (p. 100) }\end{array}$ & 95,2 & 94,0 & 93,0 & 90,6 & 96,4 & 93,2 & 93,4 & 92,2 & 97,1 & 94,6 & 95,9 & 91,6 \\
\hline $\begin{array}{l}\text { Sérum recueilli par rap- } \\
\text { port à l'eau de reconsti- } \\
\text { tution (p.100) }\end{array}$ & 32,2 & 34,8 & 40,8 & 44,0 & 19,0 & 33,5 & 31,0 & 38,9 & 13,6 & 27,0 & 19,4 & 40,8 \\
\hline $\mathrm{Ca} / \mathrm{N}$ du caillé & 0,30 & 0,33 & 0,35 & 0,38 & 0,28 & 0,30 & 0,33 & 0,36 & 0,26 & 0,28 & 0,31 & 0,33 \\
\hline
\end{tabular}


TABLEAU 9

Caractéristiques des thrombélastogrammes de mélanges de lait en poudre normal et de laits préemprésurés secs

\begin{tabular}{|c|c|c|c|c|c|c|c|c|c|}
\hline & \multirow{2}{*}{ Lait de référence } & \multicolumn{8}{|c|}{ Lait préemprésuré sec utilisé pour le mélange } \\
\hline & & $\mathrm{CN}$ & CEV 2 & CEV 3 & CUF 2 & CUF 3 & $\mathrm{PN}$ & PEV 2 & PEV 3 \\
\hline $\begin{array}{c}\mathrm{r} \\
\mathrm{mn}: \mathrm{sec}\end{array}$ & $49: 45$ & $6: 00$ & 20:30 & 22:51 & 18:18 & 8:18 & $15: 30$ & 30:39 & $32: 30$ \\
\hline $\begin{array}{c}\mathrm{k} \\
\mathrm{mn}: \mathrm{sec}\end{array}$ & 21:36 & 14:00 & 5:06 & $4: 42$ & $4: 36$ & 2:09 & $8: 45$ & $6: 33$ & $8: 30$ \\
\hline
\end{tabular}




\section{DISCUSSION}

La détermination des paramètres d'emprésurage a permis de montrer que l'utilisation de 0,5 p. 100 d'une solution enzymatique à 5 p. 100 d'une poudre de présure de force $270 \mathrm{UP} / \mathrm{g}$, convient parfaitement pour le préemprésurage à basse température, mais qu'il est préférable d'utiliser un lait préalablement concentré qui évite l'emploi de doses supérieures d'enzyme en diminuant le temps de préemprésurage et le pourcentage d'azote soluble libéré au cours de la réaction primaire (tab. 1).

Ces conclusions concordent avec les observations de différents auteurs $[21,22,23]$. Il est vraisemblable que l'accroissement de la taille des micelles et de leur instabilité favorise la coagulation et que, d'autre part, la caséine $\mathrm{K}$ des micelles est elle-même moins accessible à l'action de l'enzyme.

La température de $10^{\circ} \mathrm{C}$ utilisée pour le préemprésurage paraît la plus intéressante car elle permet une réaction enzymatique complète et suffisamment rapide sans pour autant entraîner une floculation parasite comme c'est le cas pour des valeurs plus élevées (tab. 2).

La diminution importante de la durée minimale du préemprésurage due à l'addition de calcium (tab. 3) met en évidence un démarrage précoce de la phase secondaire qui se produit bien avant la fin de la réaction enzymatique spécifique, comme l'ont déjà indiqué Cheryan et Van Wyk [24].

Les temps de préemprésurage plus importants nécessités par les laits pasteurisés normal et concentrés (tab. 4) peuvent s'expliquer par la formation de complexes entre la caséine $K$ et la $\beta$ lactoglobuline, qui freinent l'action spécifique de l'enzyme comme l'ont montré Wilson et Wheelock d'une part [25], et par une variation de l'équilibre salin qui entraîne un allongement du temps de floculation d'autre part.

Les poudres préparées par atomisation à partir des laits préemprésurés à basse température présentent des propriétés générales voisines de celles des poudres Sprays normales (tab 5). Néanmoins, nous pouvons constater une action certaine du mode de concentration des laits avant séchage. Les laits ultrafiltrés par exemple permettent d'obtenir des poudres dont la teneur en azote est très élevée et conduit à une fixation d'eau plus importante et à un rapport $\mathrm{Ca} / \mathrm{N}$ d'autant plus faible que la durée de l'ultrafiltration est importante.

Les laits concentrés par évaporation au contraire donnent des poudres dont l'humidité diminue quand le taux de concentration augmente. Il est possible que l'augmentation de la taille des micelles consécutive à l'accroissement du taux de protéines et des sels par unité de volume ou que la formation de complexes entre la caséine $\mathrm{K}$ et la $\beta$ lactoglobuline dans le cas des laits pasteurisés entraîne une dimm- 
nution d'eau liée. Cette déshydratation est certainement une des causes de l'augmentation de la teneur en azote des poudres correspondantes ainsi que de celle du calcium dans les poudres issues de de laits crus. La diminution de la concentration en calcium dans les poudres de laits pasteurisés est plus difficile à interpréter.

Les essais thrombélastographiques réalisés par reconstitution des diverses poudres préemprésurées sont caractéristiques des différents types de lait mis en œuvre $[15,16]$, mais ils mettent en évidence l'influence de la concentration et du taux de reconstitution (tab. 6). L'augmentation du temps de floculation (r) avec la concentration des laits à sécher provient du traitement thermique qui provoque un déséquilibre salin avec une baisse de la teneur en ions calcium solubles d'autant plus importante que l'opération est prolongée. Nous constatons d'ailleurs que les laits pasteurisés qui ont subi un chauffage supplémentaire présentent dans l'ensemble un allongement encore plus grand du temps de floculation. Ce même défaut de coagulation observé dans les laits ultrafiltrés de plus en plus concentrés s'explique par une fuite du calcium soluble et une chute du rapport $\mathrm{Ca} / \mathrm{N}$ (tab. 5) d'autant plus forte que l'acidification du produit est poussée et que la durée du traitement augmente (tab. 4).

L'accroissement des vitesses de floculation, de coagulation et de raffermissement du gel en fonction du taux de reconstitution est dû probablement à l'augmentation du taux de matières sèches et en particulier du calcium ionisé qui compense en partie la faible teneur en cet élément à l'origine [22].

De la même façon, l'augmentation du pourcentage de poudre de reconstitution et la rétention plus importante de l'eau d'addition qui s'ensuit sont les causes des meilleurs rendements en poids, azote et calcium constatés après égouttage dans les caillés (tab. 7).

Nous retrouvons également les effets bénéfiques de la concentration par évaporation qui, en déplaçant les équilibres salins et azotés du lait de fabrication vers la phase colloïdale entraîne un accroissement de ces mêmes rendements dans le coagulum.

A cet effet s'ajoute une action de la présure relativement moins importante dans le cas des laits concentrés qui réduit la formation d'azote soluble (tab. 1) et sa fuite lors de l'égouttage des caillés provenant de ces types de laits. Ce dernier phénomène intervient seul dans les laits ultrafiltrés et explique une augmentation plus modérée des rendements dans leur cas. Leur $\mathrm{pH}$ initial bas et l'égouttage plus prononcé qui s'ensuit sont la cause du faible pourcentage en calcium de leur caillé.

Ces résultats montrent qu'en jouant sur la concentration du lait de fabrication et le taux de reconstitution, il est possible de réduire la synérèse dans de grandes proportions; c'est ainsi que lors d'essais réalisés sur un lait concentré trois fois par évaporation et reconstitué à 33 p. 100 de poudre nous avons réussi à supprimer l'égouttage. 
L'effet de l'addition du calcium sur la synérèse semble être fonction de sa répartition entre le caillé et le sérum : suivant que la majeure partie est fixée par les protéines ou reste dans la phase aqueuse, l'égouttage est freiné ou accru. Cette hypothèse est confirmée par les valeurs du tableau 8 qui indiquent une augmentation parallèle de la fraction soluble en calcium et du volume de sérum recueilli. L'insolubilisation d'une partie du calcium permet cependant la fixation d'une plus grande quantité d'azote, ce que traduit le maintien du rendement azoté malgré un égouttage plus prononcé, et la progression du rapport $\mathrm{Ca} / \mathrm{N}$ dans le caillé.

Les résultats positifs obtenus lors de l'utilisation de poudres préemprésurées comme agents de coagulation étaient prévisibles, d'après les travaux de Cheryan et Van Wyk [24], d'autant plus que celles-ci présentent une activité résiduelle coagulante supérieure à 60 p. 100. Les effets bénéfiques constatés en outre sur la formation du caillé, par référence au lait normal, résultent simplement de l'enrichissement en matières sèches (tab. 9). Ce procédé présente enfin l'avantage de provoquer la coagulation d'un lait frais sans mettre en jeu de nouvelles quantités de présure.

Ces résultats montrent donc qu'il est possible de sécher des laits normaux ou concentrés qui ont déjà subi l'action enzymatique spécifique de la présure et que les laits secs obtenus peuvent conduire à des caillés dont l'égouttage peut être modulé ou même supprimé.

A l'intérêt nutritionnel du procédé qui permet de limiter les pertes de matières azotées, s'ajoutent de nombreux avantages économiques et techniques par rapport aux méthodes fromagères classiques tels que : l'amélioration du rendement en poids, l'économie de présure, la facilité de transport et de mise en ouvre des poudres avec des moyens technologiques simples.

L'étape suivante de nos travaux portera sur la fabrication et l'affinage de divers caillés et nous pensons qu'il sera ensuite possible de réaliser des essais complets de fabrication dans les conditions industrielles qui permettront de confirmer ces résultats préliminaires encourageants.

\section{Rés u m é}

L'emprésurage à basse température de laits ayant subi divers traitements technologiques conduit après séchage à différents types de poudres de laits préemprésurés. Après avoir défini les conditions de préemprésurage nous avons déterminé les caractéristiques physicochimiques des laits secs préparés et leur aptitude à la coagulation. Nous avons étudié leur rendement en caillé, montré qu'il était possible de maîtriser l'égouttage, et qu'ils pouvaient être utilisés comme agents coagulants. 


\section{S u m m a r y}

Cold renneting of treated milk leads to several types of milk powders which can coagulate after water has been added and the mixture has been heated. The cold renneting conditions have been specified and the physico chemical caracteristics of dry milks determined. Under certain conditions curd draining can be avoided and these powders are able to clot normal milks.

Reçu pour publication en juillet 1979.

\section{Bibliographie}

[1] I.D.F. - Monographie on Recombined dairy products. Publication prévue en 1979.

[2] FARKHONDEH (A.) (1978). - Reconstitution et recombinaison des laits et des produits laitiers. Communication au $\mathrm{XX}^{\mathrm{e}}$ Congrès international de Laiterie, Paris, juin.

[3] O'Morain (C.), Loubière (M.), Rampal (P.), Sudaka (P.) et Delmont (J.) (1978). - Etude comparative de l'insuffisance en lactase de deux populations adultes différentes (55 Niçois et 55 Maghrebins). Acta gastro-enterologica Belgica, vol. XLI.

[4] Mouchet (R.) (1978). - Rencontre internationale sur la reconstitution de fromage à partir de lait en poudre. Industries alimentaires et agricoles, 9-10, 1051.

[5] Lablée (J.) (1970). - Fabrication du fromage au lait reconstitué. Revue Laitière Française, 279, p. 599.

[6] LABLÉE (J.) (1973). - Fabrication de fromage à partir de lait en poudre et de butter oil. Technique Laitière, 790, p. 17.

[7] Maubois (J. L.), Mocouot (G.) et Vassal (L.). - Brevet d'invention du 18-7-69, $\mathrm{n}^{\circ}$ 2 502-121. Additifs des 22-10-71, 7-1-72, 28-2-73 et 28-10-74.

[8] Maubors (J. L.) et Mahaut (M.) (1974). - Application de l'ultrafiltration sur membrane dans l'industrie fromagère. Revue Laitière Française, 322, p. 479.

[9] Maubors (A.) (1978), - Les applications dans l'industrie fromagère des techniques à membranes. Communication au $\mathrm{XX}^{\mathrm{e}}$ Congrès international de laiterie, Paris, juin.

[10] Berridge (N. J.) (1952). - Some observation on the determination of the activity of rennet. Analyst., 77, 57.

[11] Sommer (H. H.) and Matsen (H.) (1935). - The relation of mastitis to rennet coagulability and curd strength of milk. J. Dairy Sc., 18, 741.

[12] Alais (C.) (1962). - Etude de l'action enzymatique de la présure sur la caséine. Isolement et composition du caséino-glycopeptide. Thèse Docteur Ingénieur, Université de Paris.

[13] HaRTeRT (H.) (1948), - Blutgerinmungstudien mit der Thrombelastographie einem neuen untersuchungsverfhren. Klin. Wochenschr., 26, 577.

[14] FrentZ (R.) (1965). - Application de la thrombélastographie de Hartert à l'étude de la coagulation du lait. Le Lait, 45, 489. 
[15] TaRodo de LA Fuente (B.) et Frentz (R.) (1966). - Etude thrombélastographique de la coagulation du lait par la présure. Action de la température et de la concentration en calcium. Le Lait, 46, 371.

[16] Tarodo de la Fuente (B.), Alais (C.) et Frentz (R.) (1969). - Etude de la coagulation du lait par la présure et de la synérèse du coagulum par la méthode thrombélastographique. Le Lait, 49, 400.

[17] LelièvRE (J.) (1977). - Rigidity modulus as a factor influencing the syneresis of renneted milk gels. J. Dairy Res., 44, 611.

[18] Linden (G.) (1971). - Applications de la spectrophotométrie d'absorption atomique dans les laboratoires d'industrie alimentaire. Industries alimentaires et agricoles, 88, 793.

[19] Serres (L.), Amariglio (S.) et Petransxiene (D.) (1973). - Contrôle de la qualité des produits laitiers. T.I. Chimie, III, $16 \mathrm{c}$.

[20] Alais (C.) (1974). - Science du lait. Edit. SEP., 164.

[21] Hostettler (H.) et ImHoF (K.) (1952). - Etude de la caséine native au microscope électronique. Ann. Agric. Suisse, 66, 307.

[22] Mocouot (G.), Alais (C.) et Chevallier (R.) (1954). - Défauts de coagulation du lait par la présure. Ann. Technol. Agric. (I.N.R.A.), 3, 1.

[23] Berridge (N. J.) (1976). - Reviews of progress of Dairy Science: continuous cheese-making. J. Dairy Res., 43, 337.

[24] Cheryan (M.) and VAn WyK (P. J.) (1975). - Secondary phase and mechanism of enzyme milk coagulation. J. Dairy Sci., 58, 477.

[25] Wilson (G. A.) and WheELock (J. V.) (1972). - Factors affecting the action of rennin in heated milk. J. Dairy Res., 39, 413. 